\title{
Hyperbilirubinemia and Follow-up Auditory Brainstem Responses in Preterm Infants
}

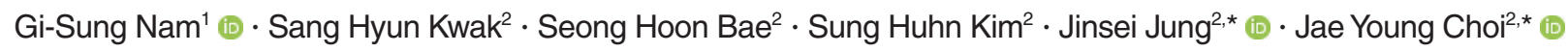 \\ ${ }^{1}$ Department of Otorhinolaryngology-Head and Neck Surgery, College of Medicine, Chonbuk National University, Jeonju;
}

${ }^{2}$ Department of Otorhinolaryngology, Yonsei University College of Medicine, Seoul, Korea

\begin{abstract}
Objectives. Neonatal hyperbilirubinemia is considered one of the most common causative factors of hearing loss. Preterm infants are more vulnerable to neuronal damage caused by hyperbilirubinemia. This study aimed to evaluate the effect of hyperbilirubinemia on hearing threshold and auditory pathway in preterm infants by serial auditory brainstem response (ABR). In addition, we evaluate the usefulness of the unconjugated bilirubin (UCB) level compared with total serum bilirubin (TSB) on bilirubin-induced hearing loss.

Methods. This study was conducted on 70 preterm infants with hyperbilirubinemia who failed universal newborn hearing screening by automated ABR. The diagnostic ABR was performed within 3 months after birth. Follow-up ABR was conducted in patients with abnormal results (30 cases). TSB and UCB concentration were compared according to hearing threshold by ABR.

Results. The initial and maximal measured UCB concentration for the preterm infants of diagnostic $\mathrm{ABR} \geq 40 \mathrm{~dB} \mathrm{nHL}$ group $(n=30)$ were statistically higher compared with $\mathrm{ABR} \leq 35 \mathrm{~dB}$ nHL group $(\mathrm{n}=40)(P=0.031$ and $P=0.003$, respectively). In follow-up ABR examination, 13 of the $A B R \geq 40 \mathrm{~dB}$ nHL group showed complete recovery, but 17 had no change or worsened. There was no difference in bilirubin level between the recovery group and non-recovery group.
\end{abstract}

Conclusion. UCB is a better predictor of bilirubin-induced hearing loss than TSB in preterm infants as evaluated by serial ABR. Serial ABR testing can be a useful, noninvasive methods to evaluate early reversible bilirubin-induced hearing loss in preterm infants.

Keywords. Hyperbilirubinemia; Bilirubin; Auditory Brain Stem Evoked Responses; Premature Infant

\section{INTRODUCTION}

Hyperbilirubinemia in infants is the major leading cause of neonatal intensive care unit (NICU) treatment and is known to be

\footnotetext{
- Received June 11, 2018

Revised August 6, 2018

Accepted September 14, 2018

- Corresponding author: Jae Young Choi

Department of Otorhinolaryngology, Yonsei University College of Medicine,

50 Yonsei-ro, Seodaemun-gu, Seoul 03722, Korea

Tel: +82-2-2228-3626, Fax: +82-2-393-0580

E-mail: jychoi@yuhs.ac

- Co-Corresponding author: Jinsei Jung

Department of Otorhinolaryngology, Yonsei University College of Medicine, 50 Yonsei-ro, Seodaemun-gu, Seoul 03722, Korea

Tel: +82-2-2228-3626, Fax: +82-2-393-0580

E-mail: jsjung@yuhs.ac

*These authors contributed equally to this work.
}

an important risk factor for neonatal hearing loss. In preterm infants, the clinical significance of relationship between hyperbilirubinemia and hearing loss is well reported [1,2]. It is also known that the auditory pathway is one of the most vulnerable area of the central nervous system for bilirubin neurotoxicity. However, the correlation between bilirubin levels and hearing outcome is still unclear. This is because bilirubin exposure cannot be accurately quantified, and auditory toxicity after exposure is also quite variable, ranging from subtle to complete hearing loss [3]. Up to $80 \%$ of preterm infants in NICU have a hyperbilirubinemia, which often present as jaundice due to bilirubin deposition. Total serum bilirubin (TSB) concentration $>25 \mathrm{mg} / \mathrm{dL}$ or above is used to determine whether phototherapy and transfusion are needed, but it has been reported that high levels of free unconjugated bilirubin (UCB) causes neuronal toxicity through the blood-brain barrier [4,5]. Bilirubin is a type of neurotoxic sub-

Copyright () 2019 by Korean Society of Otorhinolaryngology-Head and Neck Surgery.

This is an open-access article distributed under the terms of the Creative Commons Attribution Non-Commercial License (http://creativecommons.org/licenses/by-nc/4.0)

which permits unrestricted non-commercial use, distribution, and reproduction in any medium, provided the original work is properly cited. 
stance that inhibits mitochondrial enzymes and DNA synthesis, which blocks excitatory signaling of the nerves. Although bilirubin has been reported to damage cochlear nuclei and accumulation bilirubin in the auditory pathway, the mechanism of bilirubin-induced hearing loss has not been clearly established [6]. In preterm infants, precise relation between bilirubin levels and hearing threshold, including newborn hearing screening and diagnostic auditory brainstem response (ABR) is largely unknown. The purpose of this study is to evaluate the effect of hyperbilirubinemia on hearing threshold and auditory pathway in preterm infants by serial ABR. In addition, we evaluated the usefulness of the UCB concentration as compared with TSB in predicting bilirubin-induced hearing loss in preterm infants.

\section{MATERIALS AND METHODS}

\section{Patients}

All preterm infants, under 37 weeks gestational age, who were admitted to the NICU of the Severance Hospital for the treatment of hyperbilirubinemia from January 2013 to December 2017 were eligible for the study. All preterm infants were treated for hyperbilirubinemia according to the new 2007 guideline, which treatment threshold were $25 \%$ lower than previous guideline [7]. Of these, 85 infants were referred from universal newborn hearing screening (UNHS) by automated ABR (AABR) examination. Exclusion criteria included chromosomal abnormalities, TORCH (toxoplasmosis, rubella, cytomegalovirus and herpes simplex) infections, and cases where baseline testing could not be completed. So, the selected study population were 70 infants (Fig. 1). Risk factor that might be vulnerable to bilirubin-induced hearing loss (Apgar score $<5$ at 5 minutes, clinical sepsis, intraventricular hemorrhage grades 3 and $4, \mathrm{PCO}_{2}>60$ torr, $\mathrm{PO}_{2}<45$ torr, and $\mathrm{PH}<7.25$ ) were recorded for each patient during the study period. The study was approved by Institutional Review Board of the Yonsei University Severance Hospital (IRB No. 4-2018-0372). Since this is a retrospective study, no informed consent was obtained.

\section{H I G H L I G H T S}

- Serial auditory brainstem response (ABR) testing can be a useful, noninvasive methods to evaluate early reversible bilirubin-induced hearing loss in preterm infants.

- Unconjugated bilirubin is a better predictor than total serum bilirubin in preterm infants as evaluated by serial ABR.

- Our results highlight the importance of early detection and careful counselling for bilirubin-induced hearing loss of the preterm infants.

\section{Auditory brainstem response}

All NICU children in our hospital participate a two-stage AABR examination. The result of AABR was assumes an infant as passing the test when both ears were reacted to $35 \mathrm{~dB}$ nHL click sound stimulus. When the first test failed to pass, a retest was performed at intervals of 1 day or more. Infants who did not pass the AABR examination were referred to department of ENT for diagnostic ABR, considered as decision tools of hearing loss. The result of ABR was defined abnormal when the infant's hearing threshold exceed more than $40 \mathrm{~dB} \mathrm{nHL}$ in at least one ear. Those infants with hearing threshold $\geq 40 \mathrm{~dB} n H L$ at diagnostic ABR examination, performed follow-up ABR within 3 to 6 months. ABR hearing thresholds were defined as the smallest stimulus sound that can be observed with a $\mathrm{V}$ wave, ranging from $25 \mathrm{~dB} \mathrm{nHL}$ to $90 \mathrm{~dB} \mathrm{nHL}$ at $5 \mathrm{~dB} \mathrm{nHL}$ intervals. Latency of wave $\mathrm{V}$ was calculated at $90 \mathrm{~dB} \mathrm{nHL}$ sound stimulation.

\section{Bilirubin measurement}

Blood samples for the measurement of TSB and UCB were performed by neonatologists in NICU. Bilirubin concentration was examined in the first 5 postnatal days or when clinically indicated. Bilirubin measurements were performed using conventional laboratory techniques (standard colorimetric method). The UCB concentration was calculated by subtracting the direct bilirubin from the TSB concentration. Initial and serum peak level of bilirubin concentration was checked during NICU admission.

\section{Statistical analysis}

All statistical analyses were performed using SPSS ver. 12.0 (SPSS Inc., Chicago, IL, USA). The difference in parameters between two groups was tested using independent $t$-test. Spearman correlation analysis was used to identify linear associations between two variables. The sensitivity and specificity of bilirubin level as

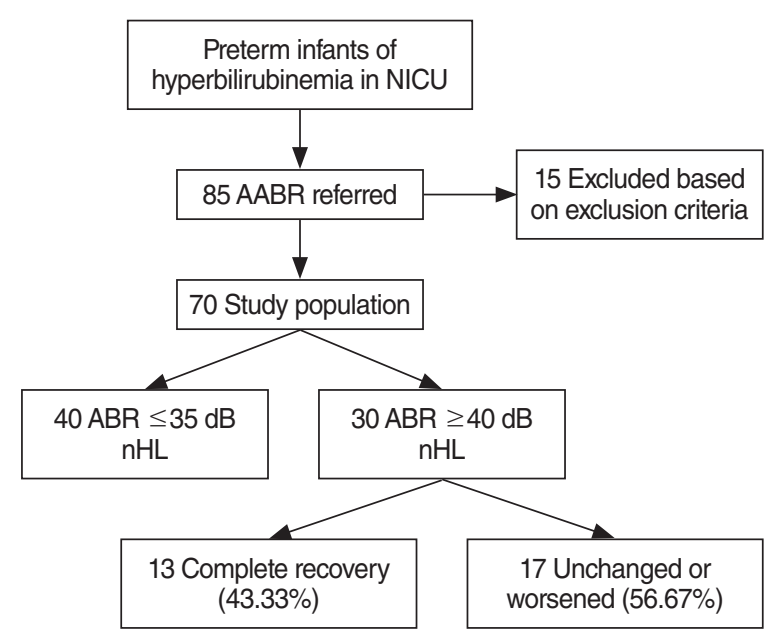

Fig. 1. Flow diagram with included and hearing characteristics of preterm infants in neonatal intensive care unit (NICU). AABR, automated ABR; ABR, auditory brainstem response. 
predictors of hearing loss were evaluated using receiver operating characteristic (ROC) curves. All data are presented as means and standard deviations. A $P$-value $<0.05$ was considered to reflect statistically significance.

\section{RESULTS}

During the study period, 85 infants who suffered from hyperbilirubinemia did not pass the newborn hearing screening and performed diagnostic ABR. Fifteen were excluded based on predefined exclusion criteria. A total 70 infants were enrolled. Flow diagram with included infants and hearing characteristics is giv-

Table 1. Clinical characteristics, risk factors of hyperbilirubinemic preterm infants $<37$ weeks of gestational age stratified by diagnostic $A B R$ thresholds

\begin{tabular}{lccc}
\hline Characteristics & Total $(\mathrm{n}=70)$ & $\begin{array}{c}\mathrm{ABR} \leq 35 \mathrm{~dB} \mathrm{nHL} \\
(\mathrm{n}=40)\end{array}$ & $\begin{array}{c}\mathrm{ABR} \geq 40 \mathrm{~dB} \mathrm{nHL} \\
(\mathrm{n}=30)\end{array}$ \\
\hline $\begin{array}{l}\text { Gestational age } \\
\text { (wk) }\end{array}$ & $31.4 \pm 4.4$ & $31.0 \pm 4.5$ & $32.0 \pm 4.2$ \\
Birth weight (g) & $1,552.1 \pm 794.8$ & $1,596.2 \pm 870.6$ & $1,495.2 \pm 694.6$ \\
Male:female & $41: 29$ & $23: 17$ & $18: 12$ \\
C-sec & $54(77.1)$ & $30(75)$ & $24(80)$ \\
Apgar score $<5$ & $8(11.4)$ & $3(7.5)$ & $5(16.7)$ \\
Sepsis & $25(35.7)$ & $15(37.5)$ & $10(33.3)$ \\
$\mathrm{PCO}_{2}>60$ torr & $4(5.7)$ & $2(5)$ & $2(6.7)$ \\
$\mathrm{pH}<7.25$ & $9(12.9)$ & $5(12.5)$ & $4(13.3)$ \\
$\mathrm{PO}_{2}<45$ torr & 0 & 0 & 0 \\
IVH $>$ II & 0 & 0 & 0 \\
Threshold of & $42.3 \pm 23.3$ & $25.6 \pm 5.8$ & $64.5 \pm 18.9$ \\
diagnostic & & & \\
ABR (dB nHL) & & & \\
\hline
\end{tabular}

Values are presented as mean \pm standard deviation or number (\%). $\mathrm{ABR}$, auditory brainstem response; C-sec, caesarean section; IVH, intraventricular hemorrhage.

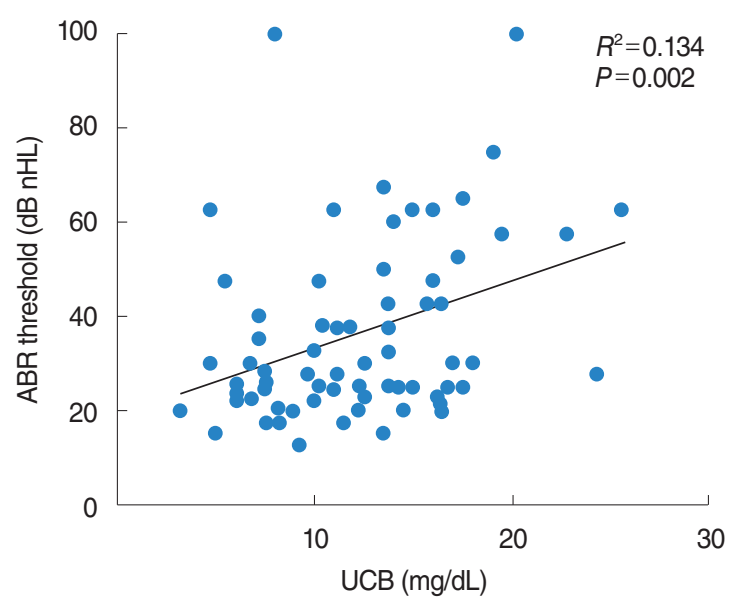

Fig. 2. Correlation between bilirubin concentration and diagnostic auditory brainstem response (ABR) threshold. (A) Relationship between unconjugated bilirubin (UCB) and ABR threshold. (B) Relationship between total serum bilirubin (TSB) and ABR threshold. en in Fig. 1. Of these 70 infants, 40 were identified as normal hearing $(\leq 35 \mathrm{~dB} \mathrm{nHL})$ and 30 were identified as abnormal hearing ( $\geq 40 \mathrm{~dB} \mathrm{nHL}$ ) in the diagnostic ABR. The follow-up ABR performed within 3-6 months showed complete recovery $(\leq 35 \mathrm{~dB} \mathrm{nHL})$ in $13(43.33 \%)$, unchanged or worsened in 17 (56.67\%) (Fig. 1). Of these 17, eight performed additional follow-up ABR within 12 months, two were recovered to normal hearing.

The clinical characteristics, risk factors of study population are given in Table 1. The mean gestational age and birthweight for the preterm infants were $31.4 \pm 4.4$ weeks and 1,552.1 $\pm 794.8 \mathrm{~g}$, respectively. The infants of the diagnostic $\mathrm{ABR} \leq 35 \mathrm{~dB}$ nHL and $\mathrm{ABR} \geq 40 \mathrm{~dB} \mathrm{nHL}$ group were no significant difference in gestational age $(P=0.337)$, birth weight $(P=0.572)$ and other risk factor (Table 1).

As shown in Table 2, preterm infants of $A B R \geq 40 \mathrm{~dB} n H L$ group showed significant differences in initial measured and maximal measured UCB concentration $(P=0.031$ and $P=0.003$, respectively) compared with $\mathrm{ABR} \leq 35 \mathrm{~dB}$ nHL group. No difference was observed in the TSB concentration at initial mea-

Table 2. Comparison of bilirubin concentration between $\mathrm{ABR} \leq 35$ $\mathrm{dB} \mathrm{nHL}$ and $\geq 40 \mathrm{~dB} \mathrm{nHL}$ groups

\begin{tabular}{lcrrr}
\hline Variable & Total & $\begin{array}{c}\text { ABR } \\
\leq 35 \mathrm{~dB} \mathrm{nHL} \\
(\mathrm{n}=40)\end{array}$ & $\begin{array}{c}\text { ABR } \\
\geq 40 \mathrm{~dB} \mathrm{nHL} \\
(\mathrm{n}=30)\end{array}$ & \\
\hline $\begin{array}{c}\text { TSB }(\mathrm{mg} / \mathrm{dL}) \\
\text { Initial measured }\end{array}$ & $10.9 \pm 3.3$ & $10.9 \pm 3.3$ & $11.0 \pm 3.4$ & 0.818 \\
$\quad$ Maximal measured & $19.6 \pm 5.1$ & $18.8 \pm 5.3$ & $20.6 \pm 4.8$ & 0.138 \\
UCB (mg/dL) & & & & \\
Initial measured & $10.6 \pm 5.1$ & $9.5 \pm 4.6$ & $12.1 \pm 5.4$ & 0.031 \\
Maximal measured & $12.5 \pm 4.8$ & $11.1 \pm 4.5$ & $14.4 \pm 4.7$ & 0.003 \\
\hline
\end{tabular}

Values are presented as mean \pm standard deviation.

$A B R$, auditory brainstem response; TSB, total serum bilirubin; UCB, unconjugated bilirubin.

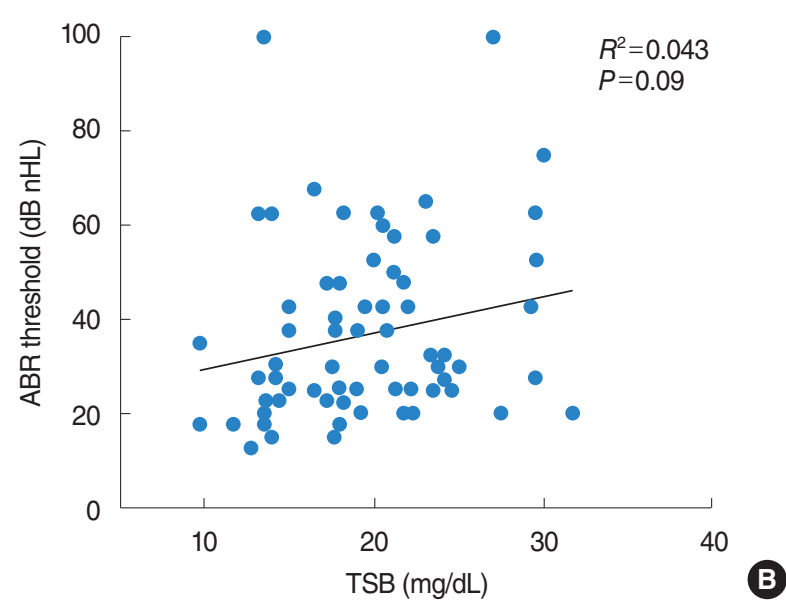


sured and maximal measured concentration (Table 2).

Maximal concentration of TSB and diagnostic ABR thresholds were positive correlated but not statistically significant $\left(R^{2}=\right.$ 0.043, $P=0.09$ ), while maximal UCB concentration and ABR thresholds were statistically significant $\left(R^{2}=0.134, P=0.002\right)$ (Fig. 2).

We plotted the ROC curves to compare TSB and UCB as a predictor of hearing loss, defined $\geq 40 \mathrm{~dB} n H L$ in diagnostic $\mathrm{ABR}$ (Fig. 3). Area under the curves of TSB and UCB were 0.591 and 0.705 , respectively. UCB is a more sensitive and specific predictor of hearing loss indicating that a UCB level of $13 \mathrm{mg} / \mathrm{dL}$ provided the best sensitivity $(70 \%)$ and specificity $(70 \%)$.

Infants who had a threshold of $40 \mathrm{~dB}$ nHL or more in diag-

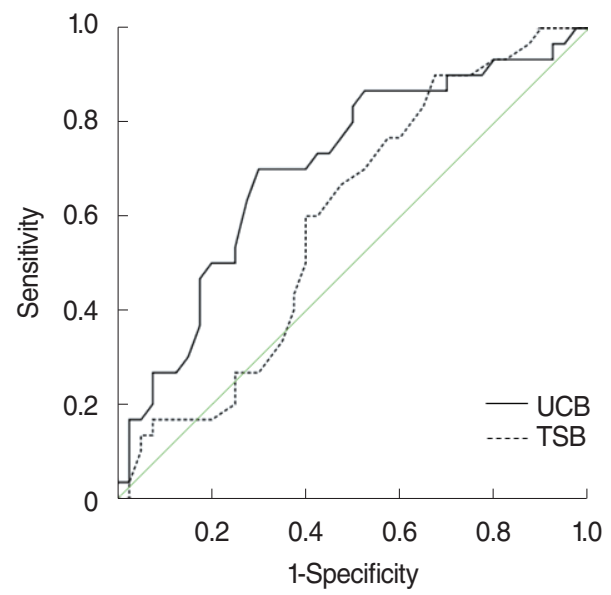

\begin{tabular}{lccccc}
\hline Variable & AUC & SE & $\begin{array}{c}\text { Asymptotic } \\
\text { sig }\end{array}$ & $\begin{array}{c}\text { Asymptomatic } \\
\text { lower bound }\end{array}$ & $\begin{array}{c}95 \% \text { Cl upper } \\
\text { bound }\end{array}$ \\
\hline UCB & 0.705 & 0.064 & 0.004 & 0.580 & 0.830 \\
TSB & 0.591 & 0.068 & 0.194 & 0.458 & 0.725 \\
\hline
\end{tabular}

Fig. 3. Receiver operating characteristic curves predicting the risk of hearing loss according to bilirubin level. UCB, unconjugated bilirubin; TSB, total serum bilirubin; AUC, area under the curve; SE, standard error; sig, significance; $\mathrm{Cl}$, confidence interval. ${ }^{a}$ Under the

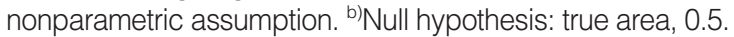

nostic ABR were divided into two groups according to follow-up $\mathrm{ABR}$ results, complete recovered group $(\leq 35 \mathrm{~dB} \mathrm{nHL}, \mathrm{n}=13)$ and non-recovered group ( $\geq 40 \mathrm{~dB} \mathrm{nHL}, \mathrm{n}=17)$. As shown in Table 3, in complete recovered group, follow-up ABR threshold was improved by about $30 \mathrm{~dB}$ nHL. However, there was no significant differences in wave $V$ latency between two groups, and there was also no significant difference in bilirubin concentration (Table 3).

\section{DISCUSSION}

Hyperbilirubinemia is one of the most common neonatal diseases that requires admission in the NICU. Severe hyperbilirubinemia in infants can cause a life-long debilitating disorder, therefore, early identification of at-risk infants during the neonatal period is crucial to promote the long-term outcome [8]. This retrospective study showed hearing results of the preterm infants of hyperbilirubinemia by serial ARB and relationship between hearing status and UCB compared with TSB.TSB concentration was considered to be a biochemical marker used for the determining the treatment, such as blood exchange transfusion or phototherapy. Many prospective studies have demonstrated association between TSB concentration and bilirubin-induced neuronal damage although there is substantial evidence that TSB concentration is a poor predictor of neuronal toxicity including hearing loss and auditory neuropathy spectrum disorder (ANSD) [9-11]. Otherwise, there is a growing evidence that free $\mathrm{UCB}$ concentration is a better indicator of auditory system damage $[1,12,13]$. Free UCB, the fraction of UCB not bound to albumin can cross the blood-brain barrier and cause neuronal damages including hearing loss. Since there is no way to measure free UCB directly in our institution, we conducted this study with only UCB concentration. While free UCB is a more sensitive marker of bilirubin-induced neuronal damage, UCB is an another sensitive marker because TSB and UCB concentration correlate well with free UCB $[12,14]$.

Table 3. Comparison of follow-up hearing results and bilirubin concentration between complete recovered and non-recovered group

\begin{tabular}{|c|c|c|c|c|c|}
\hline \multirow{2}{*}{ Variable } & \multicolumn{2}{|c|}{ Complete recovered $(n=13)$} & \multicolumn{2}{|c|}{ Non-recovered $(n=17)$} & \multirow{2}{*}{$P$-value } \\
\hline & Initial & Follow-up & Initial & Follow-up & \\
\hline $\mathrm{ABR}$ & & & & & - \\
\hline Threshold (dB nHL) & $50.2 \pm 19.1$ & $20.4 \pm 5.8$ & $57.4 \pm 24.6$ & $56.3 \pm 26.2$ & \\
\hline Wave V latency (ms) & $7.3 \pm 0.7$ & $6.7 \pm 0.4$ & $7.2 \pm 0.6$ & $6.6 \pm 0.4$ & \\
\hline \multicolumn{6}{|l|}{ TSB (mg/dL) } \\
\hline Initial measured & \multicolumn{2}{|c|}{$12.0 \pm 2.9$} & \multicolumn{2}{|c|}{$10.3 \pm 3.7$} & 0.231 \\
\hline Maximal measured & \multicolumn{2}{|c|}{$20.2 \pm 4.2$} & \multicolumn{2}{|c|}{$20.9 \pm 5.3$} & 0.182 \\
\hline \multicolumn{6}{|l|}{ UCB (mg/dL) } \\
\hline Initial measured & \multicolumn{2}{|c|}{$11.3 \pm 5.3$} & \multicolumn{2}{|c|}{$12.7 \pm 5.5$} & 0.105 \\
\hline Maximal measured & \multicolumn{2}{|c|}{$14.0 \pm 4.3$} & \multicolumn{2}{|c|}{$14.8 \pm 5.1$} & 0.474 \\
\hline
\end{tabular}

Values are presented as mean \pm standard deviation.

ABR, auditory brainstem response; TSB, total serum bilirubin; UCB, unconjugated bilirubin. 
In this study, we found that UCB concentration were significantly increased in infants with abnormal findings in diagnostic $\mathrm{ABR}(\geq 40 \mathrm{~dB} \mathrm{nHL})$ than in TSB concentration. In addition, direct correlation was found between the UCB concentration and the hearing threshold confirmed by ABR. Moreover, a significant relationship was founded between UCB level and hearing loss, which defined threshold $\geq 40 \mathrm{nHL}$ in diagnostic ABR. In ROC curves, UCB level above $13 \mathrm{mg} / \mathrm{dL}$ provide best sensitivity and specificity (Fig. 3). These results of our study and several others suggest that UCB in preterm infants related with hyperbilirubinemia is a better predictor of bilirubin-induced hearing loss as indicated by diagnostic ABR. The degree of hearing loss confirmed by diagnostic ABR is likely to be associated with UCB concentration. This was corroborated by Ahlfors and Parker [1] who reported that free UCB concentration was better predictor than TSB concentration of failed UNHS, as evaluated AABR in a retrospective study. However, compared with our study, the subject consisted of infants over 34 weeks of gestational age, not limited to preterm who were not confirmed hearing loss by diagnostic ABR. Similar to our study, in a prospective study of preterm infants, free UCB concentration was found to be more sensitive and specific predictor than TSB of abnormal ABR waveform change, but they did not identify the threshold change with follow-up ABR [12].

Bilirubin is a kind of neurotoxic substance that changes in energy metabolism, alteration in membrane function, decreased membrane potential, alteration in enzyme function, and inhibition of protein and DNA synthesis $[15,16]$. Although correlation between hearing loss and bilirubin-induced neuronal damage has been studied for a long time, type and sited of lesion are not exactly known [6]. It appears that in vitro study immature cells are more susceptible to bilirubin toxicity than differentiated cells, may also support a clinical experience that preterm infants are more susceptible to bilirubin-induced neuronal damage [17]. Bilirubin has been reported damage to cochlear nuclei and auditory pathway through cochlear nerve, and the cochlear itself is often normal [6]. Thus, hyperbilirubinemia often causes ANSD [18], which defined by abnormal ABR finding in the presence of normal optoacoustic emissions (OAE). Rance [19] reported that more than $50 \%$ of children with ANSD have a history of hyperbilirubinemia in the neonatal period. Similarly, Saluja et al. [18] found that, of the infants with hyperbilirubinemia requiring exchange transfusion, $46 \%$ had abnormal ABR wave consistent with ANSD. Although hyperbilirubinemia is commonly associated with ANSD, we did not routinely perform OAE in all cases. Eight of the 17 infants with abnormal findings in follow-up ABR performed OAE, and one infant was diagnosed with ANSD. We will continue to follow up these patients to confirm additional ANSD while performing proper auditory rehabilitation.

Congenital hearing loss should be detected within 3 months and early intervention such as hearing aids within 6 months is essential for auditory development [20]. Although UNHS has become widely used, maintaining sensitivity and specificity for appropriate screening result is still a difficult theme to consider. Neonatal otitis media with effusion is one of the significant cause of failed newborn hearing screening with reported rated $21 \%$ [21]. It can prove to be very challenging to confirm OME by pneumatic otoscope and tympanometry in neonate, even by experienced examiner. Therefore, some infants who have recovered to normal hearing in follow-up ABR are likely to have OME, and may have affect the outcome of our study.

ABR is most commonly used modality to investigate bilirubin-induced neuronal damage in neonates and preterm infants, which as a direct, noninvasive assessment. Several studies have demonstrated reversible changes in ABR wave latency. However, at the infant's periods wave I and III are not always detectable and therefore sequential measurements of latencies are not always possible [22]. In this study, only wave $\mathrm{V}$ was detectable in most cases, so wave $\mathrm{V}$ was used for analysis. In complete recovered group and non-recovered group, the wave $\mathrm{V}$ latency tended to be shorter on follow-up ABR, but, there were no significant differences between the two groups.

There is much interest in the potential reversibility of bilirubin-induced hearing loss and there is growing evidence for the reversibility of ABR abnormalities in animal models [12,23]. Mild ABR abnormalities in infants with hyperbilirubinemia may reverse with intervention by phototherapy and exchange blood transfusion. Nakamura et al. [24] found that prolonged ABR wave $\mathrm{I}$ and $\mathrm{V}$ latency resolved after exchange transfusion in a prospective study of infant with TB levels $>15 \mathrm{mg} / \mathrm{dL}$. It has been suggested that diagnostic ABR is sensitive to the earliest manifestations of neuronal damage, and treatment of hyperbilirubinemia by lowering TSB concentration at the time of abnormal ABR may reduce neuronal damage, but there have been no controlled trials to confirm this finding [25]. Hearing loss caused by hyperbilirubinemia had a recovery rate close to $80 \%$ in normal infants [22], while it was less resilient than expected in premature infants of this study. In our study, we also observed that 13 of 30 infants $(43.33 \%)$ with abnormal finding at diagnostic ABR recovered to normal hearing in follow-up test within 6 to 9 months. Of the 17 infants with abnormal finding at follow-up $\mathrm{ABR}, 12$ were able to follow-up continuously more than 1 year. Additionally, two infants recovered to normal hearing within follow-up periods, and as a result, 15 infants $(50 \%)$ with abnormal results at diagnostic ABR were recovered eventually. Therefore, we recommend that infants should be evaluated through sequential ABR more than 1 year even if they have abnormal findings on diagnostic to follow up ABR. We constantly follow up non-recovered group with hearing aids and proper rehabilitation. Hearing loss caused by hyperbilirubinemia is found to be reversible but follow-up ABR test can also show permanent hearing loss, therefore it is necessary to accurately diagnose the bilirubin-induced hearing loss by sequential ABR test. In particular, it is considered necessary to provide a more careful coun- 
seling in cases of bilirubin-induced hearing loss in preterm infants.

We acknowledge a few other limitations. First, we could not control effectively various factors related to hearing loss due to characteristics of premature infants and could not include normal control group. Secondly, we could not perform initial and followup otoacoustic emission routinely, so the possibility of ANSD cannot be excluded. Thirdly, the retrospective character of this relative small study and concomitant selection bias is insufficient to draw conclusions. Long-term implications require additional investigation.

Despite these limitations, this is the first study to report on evaluation of relationship between hearing status and bilirubin concentration through the AABR, diagnostic ABR to follow up ABR. Our study demonstrates that UCB concentration is a better predictor of bilirubin-induced hearing loss than TSB in preterm infants as evaluated by serial ABR. Serial ABR testing can be a useful, noninvasive methods to evaluate early reversible bilirubin-induced hearing loss in preterm infants.

\section{CONFLICT OF INTEREST}

No potential conflict of interest relevant to this article was reported.

\section{ORCID}

Gi-Sung Nam https://orcid.org/0000-0002-8392-5759

Jinsei Jung https://orcid.org/0000-0003-1906-6969

Jae Young Choi https://orcid.org/0000-0001-9493-3458

\section{REFERENCES}

1. Ahlfors CE, Parker AE. Unbound bilirubin concentration is associated with abnormal automated auditory brainstem response for jaundiced newborns. Pediatrics. 2008 May;121(5):976-8.

2. Mazeiras G, Roze JC,Ancel PY, Caillaux G, Frondas-Chauty A, Denizot S, et al. Hyperbilirubinemia and neurodevelopmental outcome of very low birthweight infants: results from the LIFT cohort. PLoS One. 2012;7(1):e30900.

3. Shapiro SM, Nakamura H. Bilirubin and the auditory system. J Perinatol. 2001 Dec;21 Suppl 1:S52-5.

4. Ye HB, Shi HB, Wang J, Ding DL, Yu DZ, Chen ZN, et al. Bilirubin induces auditory neuropathy in neonatal guinea pigs via auditory nerve fiber damage. J Neurosci Res. 2012 Nov;90(11):2201-13.

5. Jiang ZD, Wilkinson AR. Impaired function of the auditory brainstem in term neonates with hyperbilirubinemia. Brain Dev. 2014 Mar; 36(3):212-8.
6. Shapiro SM. Bilirubin toxicity in the developing nervous system. Pediatr Neurol. 2003 Nov;29(5):410-21.

7. van Imhoff DE, Dijk PH, Hulzebos CV; BARTrial Study Group; Netherlands Neonatal Research Network. Uniform treatment thresholds for hyperbilirubinemia in preterm infants: background and synopsis of a national guideline. Early Hum Dev. 2011 Aug;87(8):521-5.

8. Bhutani VK, Stevenson DK. The need for technologies to prevent bilirubin-induced neurologic dysfunction syndrome. Semin Perinatol. 2011 Jun;35(3):97-100.

9. Kim MH, Yoon JJ, Sher J, Brown AK. Lack of predictive indices in kernicterus: a comparison of clinical and pathologic factors in infants with or without kernicterus. Pediatrics. 1980 Dec;66(6):852-8.

10. Turkel SB, Guttenberg ME, Moynes DR, Hodgman JE. Lack of identifiable risk factors for kernicterus. Pediatrics. 1980 Oct;66(4):502-6.

11. O'Shea TM, Dillard RG, Klinepeter KL, Goldstein DJ. Serum bilirubin levels, intracranial hemorrhage, and the risk of developmental problems in very low birth weight neonates. Pediatrics. 1992 Dec; 90(6):888-92.

12. Amin SB, Ahlfors C, Orlando MS, Dalzell LE, Merle KS, Guillet R. Bilirubin and serial auditory brainstem responses in premature infants. Pediatrics. 2001 Apr;107(4):664-70.

13. Nakamura H, Lee Y. Microdetermination of unbound bilirubin in icteric newborn sera: an enzymatic method employing peroxidase and glucose oxidase. Clin Chim Acta. 1977 Sep;79(2):411-7.

14. Wennberg RP, Ahlfors CE, Bhutani VK, Johnson LH, Shapiro SM.Toward understanding kernicterus: a challenge to improve the management of jaundiced newborns. Pediatrics. 2006 Feb;117(2):474-85.

15. Cashore WJ. The neurotoxicity of bilirubin. Clin Perinatol. 1990 Jun;17(2):437-47.

16. Hansen TW. Bilirubin in the brain: distribution and effects on neurophysiological and neurochemical processes. Clin Pediatr (Phila). 1994 Aug;33(8):452-9.

17. Rhine WD, Schmitter SP, Yu AC, Eng LF, Stevenson DK. Bilirubin toxicity and differentiation of cultured astrocytes. J Perinatol. 1999 Apr-May;19(3):206-11.

18. Saluja S, Agarwal A, Kler N, Amin S. Auditory neuropathy spectrum disorder in late preterm and term infants with severe jaundice. Int $\mathrm{J}$ Pediatr Otorhinolaryngol. 2010 Nov;74(11):1292-7.

19. Rance G. Auditory neuropathy/dys-synchrony and its perceptual consequences. Trends Amplif. 2005;9(1):1-43.

20. American Academy of Pediatrics; Joint Committee on Infant Hearing. Year 2007 position statement: principles and guidelines for early hearing detection and intervention programs. Pediatrics. 2007 Oct; 120(4):898-921.

21. Holster IL, Hoeve LJ, Wieringa MH, Willis-Lorrier RM, de Gier HH. Evaluation of hearing loss after failed neonatal hearing screening. J Pediatr. 2009 Nov;155(5):646-50.

22. Shapiro SM, Popelka GR. Auditory impairment in infants at risk for bilirubin-induced neurologic dysfunction. Semin Perinatol. 2011 Jun; 35(3):162-70

23. Amin SB. Clinical assessment of bilirubin-induced neurotoxicity in premature infants. Semin Perinatol. 2004 Oct;28(5):340-7.

24. Nakamura H,Takada S, Shimabuku R, Matsuo M, MatsuoT, Negishi H. Auditory nerve and brainstem responses in newborn infants with hyperbilirubinemia. Pediatrics. 1985 Apr;75(4):703-8.

25. Smith CM, Barnes GP, Jacobson CA, Oelberg DG. Auditory brainstem response detects early bilirubin neurotoxicity at low indirect bilirubin values. J Perinatol. 2004 Nov;24(11):730-2. 